\title{
Transverse Sinus Mass Misinterpreted As The Source Of Cardiac Emboli
}

\author{
Hassan Allam ${ }^{1}$, Abdulhalim Kinsara ${ }^{2}$, Amtalkaliq Alrajawi ${ }^{1}$, Tareq Tuaima ${ }^{1}$, and \\ Shadwan Alfakih ${ }^{1}$ \\ ${ }^{1}$ King Abdullah Medical Complex Jeddah \\ ${ }^{2}$ Ministry of National Guard-Health Affairs, King Saud Bin Abdulaziz University for \\ Health Sciences, COM-WR, King Abdullah International Medical Research Center
}

June 23, 2020

\begin{abstract}
Due to the proximity of the transvers sinus (TS) to the left atrial appendage (LAA) and pulmonary veins (PV), a mass in the TS can be misinterpreted as a LAA or PV thrombus, and considered as a source of emboli in a patient with stroke or TIA . The incorrect identification of a mass as a LAA thrombus would initiate unnecessary anticoagulation therapy or potentially, an evaluation for the excision of the mass if there is a concern about dislodgement. We are presenting a case illustrating this confusion and review the literature for similar cases.
\end{abstract}

\section{Points To Learn:}

Transvers sinus fat is a differential diagnosis for a left atrium thrombus

Careful transesophageal echocardiography is a useful strategy to differentiate between transvers sinus fat and a left atrial thrombus.

\section{Introduction}

The transverse sinus (TS) is open at both ends and formed by the reflection of the visceral serosal pericardium from the posterior aspects of the aortic and pulmonary trunks over to the anterior aspect of the atrium (1). Thus, a finger in the transverse sinus will pass behind the aortic and pulmonary trunks but in front of the superior vena cava on the right and the left atrial appendage on the left (2). A Transesophageal Echocardiography (TEE) in the mid esophageal view can, in most cases, distinguish the location of a TS mass, without the need for further investigation (CT or MRI).

Case report: A 50-year-old diabetic woman presented with left sided numbness. The patient was admitted in Neurology for further investigation related to stroke of TIA. The clinical examination revealed that the patient was hemodynamically stable, with no motor weakness. The cardiac examination revealed a wide fixed second heart sound, and an ejection systolic murmur on the upper left sternum $2 / 6$. The carotid was normal. The ECG was in sinus rhythm with Right Bundle Bransh Block. The laboratory report indicated a normal CBC and renal function. The HgbA1c was 7.5 with the LDL $4 \mathrm{mmol} / \mathrm{L}$.

The TTE indicated a normal LV systolic function, normal valve, and LAVI $43 \mathrm{ml} / \mathrm{m}^{2}$. The right side of heart was dilated with the pulmonary artery pressure, with Resting PASP, $45 \mathrm{~mm} / \mathrm{hg}$. The TEE was done as a work up for stroke and revealed a mobile mass in the transverse sinus (TS). The differential diagnosis was a LAA thrombus (Figure 1A/b \& 2A/b) or PV mass (Figure 3) versus fat in the TS (Figure 3). However, 
the different mid-esophageal views assisted in the distinction of the exact location of the mass, avoiding the initiation of unnecessary interventions (figure 4).

\section{Discussion}

The incorrect identification of a mass as a LAA thrombus, would cause the initiation of unnecessary anticoagulation therapy or potentially, an evaluation for the excision of the mass if there is a concern about dislodgement. A TEE can reliably distinguish the location of a TS mass, avoiding the initiation of unnecessary investigations or interventions (3). To identify a mass (fat) in the transverse sinus, rotating the transducer will document that it is within the transverse sinus and not the LAA. Transverse sinus fat does not need any intervention for itself, but it is associated with an increased severity of coronary artery disease which necessitates more risk factor control, especially in the context of our patient being a diabetic and dyslipidemic (4). There is similar case of a transverse sinus mass mistaken as a possible aortic abscess (5).

Fig1A. Mass in transverse sinus, thought to be a LAA thrombus

Fig 1b. Mid-esophageal view clarify the mass in transverse sinus

Fig 2A. A focus LAA view showing clean LAA

Fig 2b. Clean LAA

Fig 3. Mass in TS away from PV (pulmonary vein)

Fig 4. A video view by rotating the transducer documented that it is within the transverse sinus and not the LAA

\section{References:}

1.Giove GC, Singla I, Mishra J, Nanda NC. Transesophageal echocardiographic finding of left atrial appendage lobe mimicking a mass lesion. Echocardiography 2011. Jul;28(6):684-685. 10.1111/j.15408175.2011.01438.x [PubMed ] [CrossRef ] [Google Scholar ]

2. Levy-Ravetch M, Auh YH, Rubenstein WA, Whalen JP, Kazam E. CT of the pericardial recesses. AJR Am J Roentgenol 1985. Apr;144(4):707-714. 10.2214/ajr.144.4.707 [PubMed ] [CrossRef ] [Google Scholar ]

3. Karakus G, Kodali V, Inamdar V, Nanda NC, Suwanjutah T, Pothineni KR. Comparative assessment of left atrial appendage by transesophageal and combined two- and three-dimensional transthoracic echocardiography. Echocardiography 2008. Sep;25(8):918-924. 10.1111/j.1540-8175.2008.00758.x [PubMed ] [CrossRef ] [Google Scholar

4. ] Okura K, Maeno K, Okura S, Takemori H, Toya D, Tanaka N, Miyayama S. Pericardial fat volume is an independent risk factor for the severity of coronary artery disease in patients with preserved ejection fraction. J Cardiol 2015; 65:37-41.

5.Albulushi A ${ }^{*}$ KhanF, Al-SaidiK ,Porter. T An Unusual Case of Cardiac Mass. Oman Med J. 2018 Mar; 33(2): 176-177. 


$$
\text { 退 }
$$



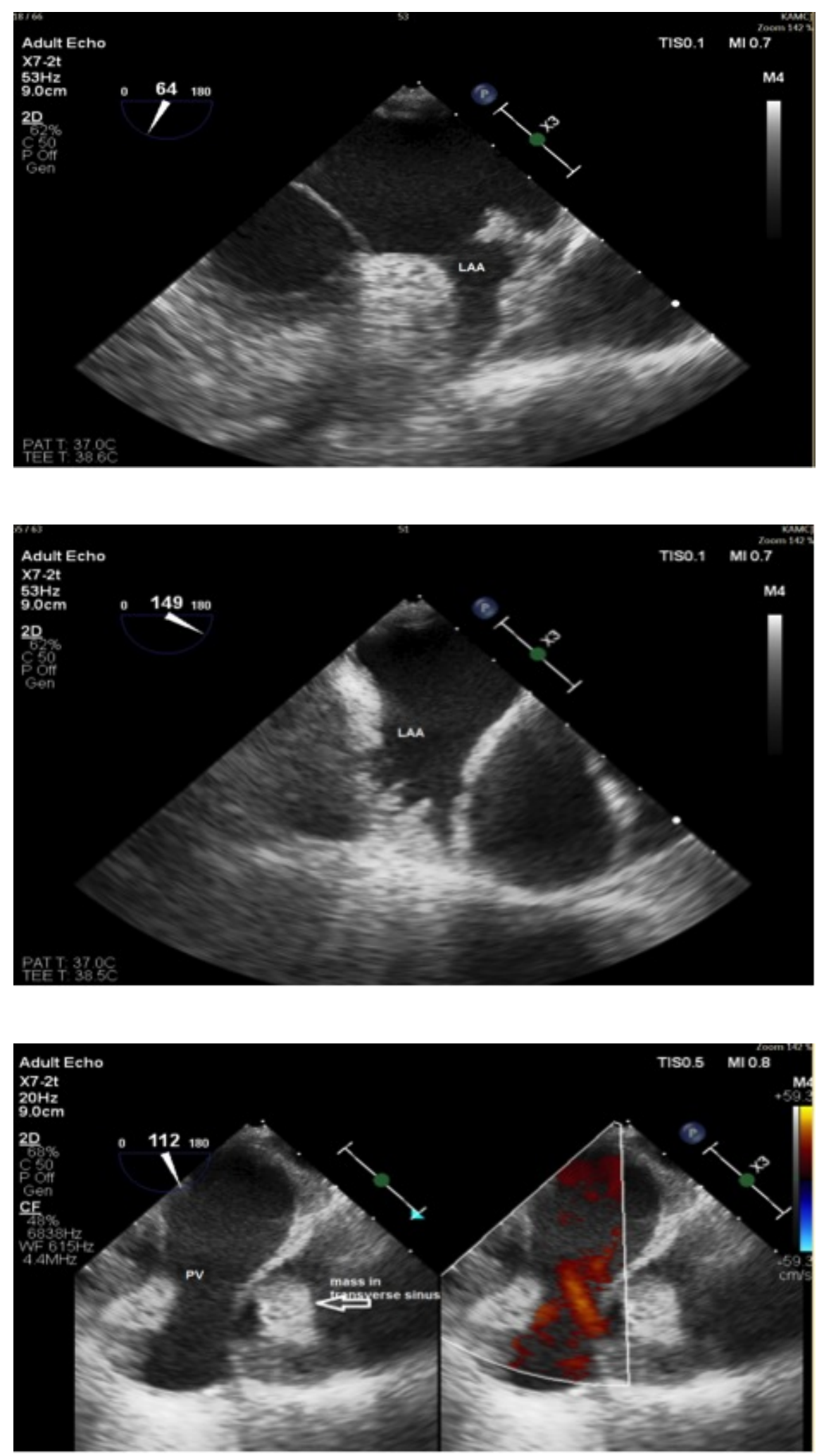

Hosted file 
laballed TS mass.mp4 available at https://authorea.com/users/336055/articles/461847transverse-sinus-mass-misinterpreted-as-the-source-of-cardiac-emboli 